\title{
Index
}

Adam, Ken 34, 87-8, 92

alcohol 110-11, 113-16

Allen, Woody 163

Andress, Ursula 131, 132, 148, 163

Aston Martin see cars

Auger, Claudine 132, 136

Austin Powers films 184

Avengers, The 137, 184

Bach, Barbara 171, 172, 196n.23

bachelor

Bond as 11

pad 87-8, 162

Baker, Stanley 63

Balio, Tino 36, 109

Baron, Cynthia 93

Basinger, Kim 176

Beatles, The 35, 184

Beats, The 27-8

Bennett, Tony 2-3, 21, 24, 35, 36, $37,63-4,65,80,122-3$, 139-40, 141, 142-3, 145, $152,165,170,179$

Benson, Raymond 41, 52, 185-7

Bergonzi, Bernard 18, 29

Beswick, Martine 136, 142

Bianchi, Daniela 132, 135, 136, 143

Black, Jeremy 16, 88, 150, 182

Blackman, Honor 134, 135, 137, 149

'Blast From the Past' 185-6

Bochenko, Irka 173

\section{Bond, James}

as icon(ic) 2, 7, 20-1, 24, 27, 58, 99, 117, 144, 159, 189, $190-1,193$

body $62,146-7,191-2$

sexuality 10, 16, 63, 144-54, 155, 177,182

Bond girl

casting of 131-2, 174-5

formula 153, 154, 169

as label 136, 176

as male fantasy $122-3,130-1$, $141,144,145$

naming of by Ian Fleming 133-4

as safeguard 146-54

use in publicity $132,134-6,163$, $171-4$

Boothroyd, Geoffrey 42

Bouchet, Barbara 163

Brabazon, Tara 128-9

Bradbury, Ray 26, 39

Breazeale, Kenon 12

Broccoli, Albert 33-4, 35, 61-2, $132,169,175$

Brosnan, Pierce 41, 183, 189-90, 192

Buchan, John 9

Caine, Michael 62-3, 161, 193

Cannadine, David 17

cars 85-6, 88-90 
Casino Royale (film 1967) 163

Casino Royale (film 2006) 188-9, 191-2

Casino Royale (novel) 10-11, 49, 55 n.58

Casino Royale (television) 33

Cawelti, John and Bruce Rosenberg 69

Chapman, James 3, 10, 20, 33, 34, 35-6, 63, 85, 144, 149, 177, 195 n.6

Chicago 14, 44-7

Chiles, Lois 173, 176

Clery, Corinne 173

clubland hero(es) 9-10, 63, 76

Coburn, James 160

Cohan, Steven 87

Conekin, Becky 98-9

conformity, playboy attitude towards $2,19,27,68-71,146$

Connery, Sean 5, 39-41, 51-2, 58-81, 84, 86, 99-100, 106, 109-10, 117, 132, 146-7, 148, 154, 161, 183, 189, 190-1, 192, 193

consumer culture

male consumerism 11-12, 75-6, 77-8, 84-117, 122, 144-5

post-war rise of $8,19-20$

Cook, Pam 184

cooking, as masculine activity 79

Cooper, Gary 63

Cossey, Tula 173-4

Craig, Daniel 188-93, 194

Curtis, Tony 178

D'Abo, Maryam 176, 188

Dahl, Roald 92, 168-70

Dalton, Timothy 181-3, 189-90

Deighton, Len 160-1

Dench, Judi 183, 186

Denning, Michael 18-19, 78, 93, 95,144
Diamonds Are Forever (film) 80, 89, $166,167-8$

Die Another Day 188-9

DoubleShot 41, 186, 197n.64

Doyle, Arthur Conan 13, 26

Dr No (film) 35-6, 40, 58-9, 62, 70, 87, 96, 102, 110-11, 126-7, 128,193

Honey Ryder in 130-1, 140, 143, $148-9,154$

Dr No (novel) 17-18, 29

Dyer, Richard 146-7

Eaton, Shirley 135, 137

Eco, Umberto 11

Ehrenreich, Barbara 19-20, 27, 72, $122,146,153,181$

Esquire 11-13, 14, 25, 26-7, 45, 84, 122

fashion 98-106, 190-1

feminism 129, 142, 171-2, 177, 180

Finney, Albert 62-3

Flint films 160-2

food 79, 111-13

For Your Eyes Only (film) 173-5

From Russia with Love (film) 40, 50, $59,85,97,111$

Red Grant in 73-4

Tatiana Romanova in 143-4, 150-1, 154

From Russia with Love (novel) 32, 97, 112-13

Fleming, Ian

book sales 21, 28-9, 36-7, 51 compared with Bond 15-16, 49 criticism of writing 17-18, 29 description of Bond 39, 61, 76-7 at Goldeneye 48, 52, 95 and Playboy 24-5, 29-31 novels serialised 37-9, 40-1, 50-2 


\section{Index}

'Playboy Interview' 32, 48-9, 91-2

as Playboy role model 52 response from 'Dear Playboy' readers' letters $31,41-3$, 49-50

use of literary celebrity $5,25-8$, $44-5,47-8,96$

visit to Playboy 44-6

and the spy thriller 9-11, 21, 63, 165

Fraterrigo, Elizabeth 13, 156n.38, 182

Gable, Clark 63

gadgets/gadgetry 85-8, 163, 167, 179, 183

Getty, J. Paul 67-8, 71-3

GoldenEye (film) 182-3, 190

Goldfinger (film) 60, 78, 85-6, 99, 108, 113-14, 115-16, 137-8, 165-6, 168

Auric Goldfinger in 88, 102-3

Pussy Galore in 134, 137, 149

Goldfinger (novel) 29-30

golf 77-8, 103, 105

Grant, Cary 2, 30, 61

grooming 107-9

Gunelius, Susan 183, 197n.44

Hagopian, Kevin J. 165

Halberstam, Judith 88

Hama, Mie 168

Hefner, Hugh 2

attitude towards sex(uality) 16-17, 133, 146, 158n.63, 180

as editorial voice for Playboy 13, 28, 66-7, 75

and Esquire 11-13, 14, 25

public image as Mr Playboy 15, 182-3, 187

Helm films 160-3

Hemingway, Ernest 25, 27

Hendry, Gloria 175-6

'Hildebrand Rarity, The' (story) 24, $30-1,38$
Hitchcock, Alfred 30, 45, 79

homosexuality, fears of 12, 146, 155

Howard, Trevor 61

Hui, Christina 173

individualism 65-74

intertextuality 3, 35, 63-4, 80-1, 141,184

Ipcress File, The see Palmer films

Istanbul 96-7

Jamaica 47-8, 95-6, 119n.39

Japan 92-5

Johnson, Paul 17-18, 29, 43

Jones, Grace 176

Kaeppler, Chichinou 173

Kennedy, John F. 2, 32, 53-4n.27

Kerouac, Jack 27

King, James 92-3

Kinsey Reports 16-17

Korda, Alexander 33

Ladenson, Elisabeth 134

Lavi, Daliah 163

Lazenby, George 56n.98, 166, 177

Leach, Jim 189

Lee, Bernard 40, 70

Lee-Hsu, Diane 176

leisure

Playboy and 66-7, 75-6, 78

see also bachelor pad

Lenya, Lotte 50

Licence to Kill (film) 181-2

Life 32, 137

Live and Let Die (film) 177, 179

Live and Let Die (novel) 29

Living Daylights, The (film) 181, 182

Lowell, Carey 176

Lycett, Andrew 29, 55n.51

McCallum, David 101

McClory, Kevin 132 
McGoohan, Patrick 61

McNeile, H. C. (Sapper) 9

Maibaum, Richard 34, 35, 132, 147-9, 151-3, 153-4

Mallet, Tania 135

Man from U.N.C.L.E, The 101, 160, 184

Man with the Golden Gun, The (novel) $37,40,42,50$

marriage, anti- 13, 15, 145-6, 152-3

see also conformity

Martin, Dean 160

Martini see alcohol

masculinity 61-3, 88, 108-9, 182 playboy model of 14

post-war 19-20, 63, 117n.1, 122, 146

see also consumer culture

Maxwell, Lois 126-8, 136

men's magazines, compared to

Playboy 180

see also Esquire

'Midsummer Night's Doom' (story) 186-7

Miller, Toby 88, 147, 169

Mills, John 62-3

Mills, Kim 173

Monroe, Marilyn 13, 123

Moonraker (film) 173, 178-9

Moonraker (novel) 106, 111, 126

Moore, Roger 76, 173, 175, 176-9, 181-2

Mulvey, Laura 142, 146

Nadel, Alan 32, 75

Never Say Never Again 80, 176, 188

Niven, David 61, 76, 163

Nolan, Margaret 135, 136

Octopussy (film) 180, 188

'Octopussy' (story) 37, 41, 52

office

Miss Moneypenny and 126-9

Playboy office 45
Playmate 124-6

relationship between Bond and $\mathrm{M}$ in 70

On Her Majesty's Secret Service (film) 89, 152-3, 166-7

On Her Majesty's Secret Service (novel) 37, 38, 39, 51, 126

Orient Express 97-8

Osgerby, Bill 3, 20, 25, 117n.1

Our Man Flint see Flint films

Palmer films 160-1

Paluzzi, Luciana 136

Paul, Art 37-8, 55n.52

Pearson, John 16, 52

Pendergast, Tom 25, 27

Peters. Molly 136, 138, 141

Pfeiffer, Lee 106

Pilgrim, Janet 124 6, 129, 133

pin-up 12, 123

Pitzulo, Carrie 12, 155n.4, 158n.63, 180

Plain, Gill 63

Playboy

bed 86-7, 162

in the Bond films 166-8

clubs 46-8, 167, 181

Enterprises 181, 182, 183

magazine

attitudes to work and play in $14,28,66-7,86-7$

'Be a James Bond girl' contest $174-5$

Bond spoofs in 160-5

circulation of 13, 21, 180

'Playboy Advisor' 116-17

'What Sort of Man Reads

Playboy?' campaign 75-6, 89

women of Bond pictorials in 6 , 134 44, 146-7, 154-5, 156-7n.38, 163, 168-76, 179,188

Mansion 15, 86, 182, 187 


\section{Index}

Playmate

girl-next-door image of 12 ,

123-4, 132-3

as male fantasy 124-5, 129-30

see also office; Pilgrim, Janet

pornography 18-19, 144

'Property of a Lady, The' (story) 37

Quantum of Solace 189, 191, 192

Ratoff, Gregory 33, 163

Regin, Nadja 135, 136

Remington Steele 190

Saint, The 20, 178, 184

Saltzman, Harry 33-4, 35, 61, 160, 169

Sellers, Peter 163

Serre, Catherine 173

sexuality, changing attitudes towards 8, 16-19, 144-5

see also Bond; Bond girl; homosexuality; Playmate

Shail, Robert 161

Silencers, The see Helm films

Sinatra, Frank 2, 48

Skyfall 89, 186, 189, 191, 192, 193

Snelling, O. F. 9-10

social mobility 75-8, 106

Spectorsky, A. C. 26, 78, 96

Spectre 89, 189, 191, 194

Spicer, Andrew 63, 183

Spy Who Loved Me, The (film) 178

Anya Amasova in 171-2, 177

Stallone, Sylvester 182

Storey, John 184

Street, Sarah 35

suit see fashion
Thrilling Cities 44 6, 47, 91-2

Thunderball (film) 58, 64, 86, 89, 100, 102, 109, 111, 132, 136, 141-2, 157n.44, 168

Emilio Largo in 103-5, 114, 148

Fiona Volpe in 151-2

Patricia Fearing in 138-9

travel 91-8

United Artists (UA) 34-5, 36, 59, 109, 174

Usborne, Richard 9

View to a Kill, $A$ (film) 181

Wakabayashi, Akiko 168

Warville, Lizzie 173

Watts, Steven 15, 182

Weinstein, Sol 164

Willis, Bruce 182

Wood, Lana 175

Woollacott, Janet 2-3, 21, 24, 35, $36,37,63-4,65,80,122-3$, 139-40, 141, 142-3, 145, $152,165,170,177-8,179$

Wright, Maggie 135, 137

Yates, Dornford 9

You Only Live Twice (film) 56n.98, 80, 92-4, 112, 114-15, 168-70

You Only Live Twice (novel) 37, 41, 42, 43, 50, 76-7, 81n.5, 92, 185

Young, Robbin 175

yuppie 181

Zero Minus Ten 186 